ESAIM: PROCEEDINGS AND SURVEYS, December 2014, Vol. 47, p. 75-96

F. Abergel, M. Aiguier, D. Challet, P.-H. Cournède, G. Fä̈, P. Lafitte, Editors

\title{
THE RESISTANCE OF THE RESPIRATORY SYSTEM, FROM TOP TO BOTTOM $^{*, * *}$
}

\author{
BERTRAND MAURY ${ }^{1}$
}

\begin{abstract}
This paper proposes different levels of modeling around the notion of resistance: from the single parameter that is commonly measured in medical practice, to more sophisticated settings that account for the geometrical characteristics of the respiratory tract.

Résumé. Cet article propose différents niveaux de modélisation permettant de donner un sens à la notion de résistance de l'arbre bronchique: de l'approche macroscopique qui définit cette résistance comme un nombre unique, mesurable, permettant de quantifier les effets résistifs, à des cadres formels plus sophistiqués prenant en compte les multiples paramètres géométriques intervenant dans ces phénomènes dissipatifs.
\end{abstract}

\section{INTRODUCTION}

The human respiratory system certainly earns the right to be called a complex system, for various reasons:

(1) The number of its constitutive elements is huge: around 16 million branches for the respiratory tract, and 300 million of alveoli (see e.g. [24]);

(2) A great variety of physical phenomena is involved in the overall respiratory process: fluid mechanics, advection, diffusion, surface tension together with macromolecule recruitment (surfactant), complex chemical reactions (oxygen binding with hemoglobin), structural mechanics of heterogeneous media, to cite a few;

(3) A wide range of orders of magnitude are involved: the typical size of the lungs is about $20 \mathrm{~cm}$, the trachea diameter is $2 \mathrm{~cm}$, for smaller branches it drops down below the millimeter, alveolar size is about a quarter $\mathrm{mm}$, the alveolocapillary membrane (that separates each alveolus from capillary vessels) is about a micrometer thick, while the red blood cell diameter is about $8 \mu \mathrm{m}$.

We shall focus here on a very particular aspect, still quite significant in medical diagnosis, namely the airway resistance, and its relation with geometric characteristics of the respiratory tract.

In the context of pneumology, the airway resistance $R_{a w}$ is essential in characterizing the patient state in terms of ventilation capability. It quantifies the relationship between the air flow rate through the respiratory tract and the pressure drop between its ends: atmospheric pressure at one end, and alveolar pressure at the other end. The first attempt to quantify this phenomenon by a single parameter was proposed a century ago by Fritz Rohrer [20], and since then it has been used intensively in the context of medical practice, or mathematical modeling (see e.g. $[1,9,13,19]$ ).

* The author would like to thank C. Grandmont, H. Guénard, A. Janon, and S. Martin, for fruitful discussions and suggestions

** This work was partially funded by the ANR project OxHelease ANR-11-TECS-006

1 Laboratoire de Mathématiques d'Orsay (LM-Orsay), CNRS : UMR8628 - Université Paris XI - Paris Sud

\& POPIX (INRIA Saclay - Ile de France) Inria

(C) EDP Sciences, SMAI 2014 
We aim here at investigating the connections between the functionalist (or top-down) standpoint, which consists in accounting for all resisting phenomena by a single parameter, directly accessible to measurment, and the physiologist (bottom-up) one, which aims at recovering the "big picture" starting from the finest scale.

The objective of the approach we propose here is twofold:

(1) Develop and describe mathematical frameworks to support and enrich the notion of resistance, and investigate its level of complexity far beyond the single parameter notion that is considered in medical practice;

(2) show that the very tree structure of the respiratory tract provides robustness and stability with respect to the huge number of involved parameters, thereby supporting it and, in some way, legitimating the use of lumped (i.e. oversimplified) models to describe the dissipation phenomena that occur during ventilation.

Section 1 is dedicated to the top-down approach, it describes how the resistance can be defined "from the outside", in the framework of a simple model based on the sole global volume. In Section 2 we take the reverse standpoint: the notion of resistance is defined for a general domain, and then computed for a cylindrical pipe (Section 2.1). The latter is used as a basic ingredient to build the notion of resistive network (2.2). A link with the macroscopic resistance is made, and an actual computation of this global resistance is proposed in Section 2.3 for a dyadic tree. The next two sections propose alternative standpoints: the dyadic integer setting, that makes it possible to perfom some kind of harmonic analysis on the set of ends of the tree, and a stochastic setting that describes the deep link between the Darcy like equations set on a network and an underlying Markov process. A fast algorithm to compute the global resistance of a dyadic tree is proposed in Section 2.6. The next section addresses optimality and stability issues related to the notion of resistance, from the standpoint of constrained optimization (Section 3.1), and from a statistical standpoint (Section 3.2).

\section{TOP-DOWN STANDPOINT: GLOBAL RESISTANCE OF THE RESPIRATORY SYSTEM}

The simplest mechanical model for ventilation is based on the volume $V$ of air contained in the lung. It is based on the following considerations: the lung is represented as a single balloon, connected to the outside world by a pipe. The pressure within the balloon is denoted by $P_{a}$ (the letter $a$ stands for alveolar pressure). The pressure outside the balloon, $P(t)$, accounts for muscular efforts (contraction of the diaphragm for inspiration, and possibly of the abdominal and intercostal muscles during forced expiration). The volume $V$ of air in the balloon is assumed to spontaneously relax toward an equilibrium value $V_{0}$. In the linear setting, the actual difference $V-V_{0}$ is related to the pressure drop by

$$
E\left(V-V_{0}\right)=P_{a}-P .
$$

The parameter $E$ is called the elastance, it quantifies the pull-back forces within the whole system.

As for the pipe, assumption is made that the flow rate is proportional to the pressure drop between the inlet of the pipe and the balloon. Setting the atmospheric pressure at 0 and assuming incompressibility of the air in the considered regime, we obtain

$$
0-P_{a}=R \times \text { flow rate }=R \dot{V} .
$$

The auxiliary variable $P_{a}$ can be eliminated, and we finally have

$$
R \dot{V}+E\left(V-V_{0}\right)=-P(t)
$$

that is a first order linear equation for the volume $t \mapsto V(t)$, with two constant parameters $E$ (elastance) and $R$ (resistance), and with a time-dependent forcing term $P(t)$.

The airway resistance $R$ can be measured experimentally, in a way that is conditioned by this very model. Let us mention here a simple way to perform it in practice, according to the so-called interrupter method. This method (see e.g. [19]) is based on a sudden interruption of airflow during spontaneous breathing. It is considered 
that during a short time after the interruption, the pressure at the mouth balances with the alveolar pressure $P_{a}$. This interruption is caused by the closure of a valve. The resistance is then deduced from Formula (1):

$$
0-P_{a}=R \dot{V}
$$

where $\dot{V}$ is the flow rate at the time of occlusion of the valve (more precisely right before this interruption, since it drops down to zero afterwards). Such measurements lead to values typically between 1 and 2 , expressed in the unit that is standard in this context, i.e. $\mathrm{cm} \mathrm{H}_{2} \mathrm{O} \mathrm{s} \mathrm{L}^{-1}=\mathrm{hPa} \mathrm{s} \mathrm{L}^{-1}$.

Further extensions. There is a huge literature dedicated to extensions / improvements of this simplistic model. As far as the resistance is concerned, the linearity of the relation between the flow rate and the pressure jumps can be ruled out. Actually, the nonlinear dependence of the flux with respect to the pressure jump was already mentioned in the 1915 seminal paper [20]. It should be stressed that the very definition of the resistance, as we presented it, is challenged by this nonlinear setting. As soon as the relation involves more than one parameter (as in the quadratic case), the notion itself becomes nebulous. We refer to [16, 18] for a detailed account of extra factors that may affect the overall resistance, but we shall restrict ourselves here to the linear model that is based on a unique and constant parameter.

Outcomes of the linear model. Despite its simplicity, this model fairly reproduces the ventilation process in the normal regime, and it allows to investigate how the process can be affected by the perturbation of the parameters. We end this section by describing straightforward (but meaningful) outcomes of the linear model. Consider the typical situation of a $T$-periodic forcing scenario (see [16] for further details)

$$
P(t)=\mid \begin{aligned}
& P_{i n s p}<0 \quad \text { in } \quad\left[0, T_{\text {insp }}[\right. \\
& P_{\text {exp }} \geq 0 \quad \text { in } \quad\left[T_{\text {insp }}, T[\right.
\end{aligned}
$$

where $T$ is the ventilation period, and $T_{\text {insp }}<T$ is the duration of the inspiration phase. No matter what the initial condition is, the volume $V(t)$ converges to a periodic function. The gap between the minimal and maximal volume is called the Tidal Volume, it quantifies the efficiency of the ventilation process in terms of oxygen renewal (and carbon dioxide evacuation). It can be expressed as

$$
V_{T}=\Lambda\left(T, T_{i n s p}, \lambda\right) \frac{P_{e x p}-P_{i n s p}}{E}
$$

where $\Lambda$ is a dimensionless constant that accounts for resistance limitation effects. It expresses as

$$
\Lambda\left(T, T_{i n s p}, \lambda\right)=\frac{\left(1-e^{-\lambda T_{i n s p}}\right)\left(1-e^{-\lambda\left(T-T_{i n s p}\right)}\right)}{1-e^{-\lambda T}}
$$

where $\lambda=E / R$ is the time constant. This expression assesses the high robustness of the process with respect to resistance variations. In the normal regime, $\tau=R / E$ is of the order $0.5 \mathrm{~s}, T_{\text {insp }} \approx 2 \mathrm{~s}, T \approx 5 \mathrm{~s}$, so that $\Lambda$ is very close to 1 , in a robust way. Indeed, $\Lambda$ considered as a function of the sole resistance $R$ is very flat in the neighborhood of the standard value for $R$ (that is between 1 and $2 \mathrm{~cm} \mathrm{H}_{2} \mathrm{O} \mathrm{s} \mathrm{L}^{-1}$ ). It can be shown straightforwardly that all the derivatives of $\Lambda$ with respect to $R$ vanish at $R=0$ (see again [16]).

\section{BotTOM UP STANDPOINT}

We aim now at describing more formally the dissipation phenomena that occur within the respiratory tract. We shall present here two sets of partial differential equations for which a notion of resistance can be defined. The Stokes equations describe the actual motion of the air in small pipes, and they are the theoretical basis of dissipative phenomena within the respiratory tract (Poiseuille's law). Besides, the Darcy equations describe 
the motion of a fluid in a porous medium. The latter do not directly describe the air flow in the respiratory tract, but they are formally very close to the resistive network system that we shall obtain from Poiseuille's law. Let us stress that we disregard here inertial effects. As a consequence, neither the partial differential equations (Stokes and Darcy) that we shall consider, nor the resulting discrete problems on networks, involve any time-derivative; they all express instantaneous force balance rather than the full Newton's law.

\subsection{Fluid models}

The general setting is the following: we consider a domain $\Omega$, the boundary $\Gamma$ of which is decomposed in three components (not necessarily connected):

$$
\Gamma=\Gamma_{\text {in }} \cup \Gamma_{\text {out }} \cup \Gamma_{w},
$$

that are respectively the inlet, the outlet, and the lateral walls. We consider that the domain is filled with a viscous fluid (viscosity $\mu$ ). The so-called Pressure Drop Problem problem reads:

$$
\left\{\begin{aligned}
-\mu \Delta \mathbf{u}+\nabla \mathbf{p} & =0 & & \text { in } \Omega, \\
\nabla \cdot \mathbf{u} & =0 & & \text { in } \Omega, \\
\mathbf{u} & =0 & & \text { on } \Gamma_{w}, \\
\mu \nabla \mathbf{u} \cdot \mathbf{n}-\mathrm{p} \mathbf{n} & =-P_{\text {in }} \mathbf{n} & & \text { on } \Gamma_{\text {in }}, \\
\mu \nabla \mathbf{u} \cdot \mathbf{n}-\mathrm{p} \mathbf{n} & =-P_{\text {out }} \mathbf{n} & & \text { on } \Gamma_{\text {out }} .
\end{aligned}\right.
$$

The boundary conditions on $\Gamma_{\text {out }}$ and $\Gamma_{\text {in }}$ are called free outlet conditions, although they concern inflow as well as outflow. They express the asumption that the outside medium (upstream $\Gamma_{\text {in }}$ and downstream $\Gamma_{\text {out }}$ ) are both set at a given pressure, which balances with the normal stress on both boundaries.

Definition 2.1. (Resistance of a domain (Stokes setting))

Let $\mathbf{u}$ be the velocity field that solves Problem (5). The flux $Q$ is defined as

$$
Q=-\int_{\Gamma_{\text {in }}} \mathbf{u} \cdot \mathbf{n}=\int_{\Gamma_{\text {out }}} \mathbf{u} \cdot \mathbf{n}
$$

By linearity of the Stokes equations, this flux linearly depends on the pressure drop $P_{\text {in }}-P_{\text {out }}$, and the resistance $R=R(\Omega)$ between $\Gamma_{\text {in }}$ and $\Gamma_{\text {out }}$ is defined by

$$
P_{\text {in }}-P_{\text {out }}=R Q
$$

This resistance can be defined in a variational way. Let us define $K$ as

$$
K=\left\{\mathbf{v} \in H^{1}(\Omega)^{n}, \mathbf{v}_{\mid \Gamma_{w}}=0, \nabla \cdot \mathbf{v}=0, \int_{\Gamma_{i n}} \mathbf{v} \cdot \mathbf{n}=-1\right\},
$$

where $n$ is the space dimension. The resistance is then defined (see [16]) as

$$
R=\inf _{\mathbf{v} \in K} \mu \int_{\Omega}|\nabla \mathbf{u}|^{2} .
$$

Resistance of a domain (Darcy).

The notion of resistance can also be defined in a Darcy setting. This approach is very similar to the way we will define it for a resistive network. The situation is the following: the domain $\Omega$ is considered as a porous 
medium, in which the (Darcy) velocity is defined as $\mathbf{u}=-k \nabla \mathrm{p}$, where $\mathrm{p}$ is the pressure, and $k$ the permeability of the medium.

$$
\left\{\begin{aligned}
\mathbf{u}+k \nabla \mathrm{p} & =0 & & \text { in } \Omega, \\
\nabla \cdot \mathbf{u} & =0 & & \text { in } \Omega \\
\mathbf{u} \cdot \mathbf{n}=-k \frac{\partial \mathrm{p}}{\partial n} & =0 & & \text { on } \Gamma_{w} \\
\mathrm{p} & =P_{\text {in }} & & \text { on } \Gamma_{\text {in }}, \\
\mathrm{p} & =P_{\text {out }} & & \text { on } \Gamma_{\text {out }} .
\end{aligned}\right.
$$

The flux $Q$ and the resistance $R$ are then defined as previously (equations (6) and (7)), so that

$$
P_{\text {in }}-P_{\text {out }}=R Q=R \int_{\Gamma_{\text {out }}} \mathbf{u} \cdot \mathbf{n}=R \int_{\Gamma_{\text {in }}} k \frac{\partial \mathrm{p}}{\partial n} .
$$

A standard Poisson problem on the pressure is obtained by eliminating the velocity.

$$
-\nabla \cdot k \nabla \mathrm{p}=0
$$

with Neuman boundary conditions on $\Gamma_{w}$, and Dirichlet B.C.'s on $\Gamma_{i n}$ and $\Gamma_{\text {out }}$. The energy balance is obtained by multiplying Poisson's equation by p, and using Green's formula:

$$
0=\int_{\Omega} k|\nabla \mathrm{p}|^{2}-\int_{\Gamma_{\text {in }}} k \frac{\partial \mathrm{p}}{\partial n} \mathrm{p}-\int_{\Gamma_{\text {out }}} k \frac{\partial \mathrm{p}}{\partial n} \mathrm{p}=\int_{\Omega} k|\nabla \mathrm{p}|^{2}+P_{\text {in }} \int_{\Gamma_{\text {in }}} \mathbf{u} \cdot \mathbf{n}+P_{\text {out }} \int_{\Gamma_{\text {out }}} \mathbf{u} \cdot \mathbf{n},
$$

thus the rate of dissipated energy within the porous medium is

$$
\mathcal{P}=\int_{\Omega} k|\nabla \mathrm{p}|^{2}=\left(P_{\text {in }}-P_{\text {out }}\right) Q=\frac{1}{R}\left(P_{\text {in }}-P_{\text {out }}\right)^{2}=R Q^{2},
$$

which mimics in this mechanical setting the famous Joule's law for electric wires : $\mathcal{P}=R I^{2}$ (where $I$ is the electric current).

Poiseuille's flow, resistance of pipes and networks.

In the case where $\Omega$ is a circular cylinder of length $L$ and diameter $D$, Problem (5) can be solved exactly (parabolic profile), and the analytic expression of the resistance is obtained:

$$
R=\frac{128 \mu}{\pi} \frac{L}{D^{4}}
$$

We consider now a network made of three of such pipes (see Fig. 1). Assuming the lengths of the pipes are significantly larger than their diameters, it is reasonable to expect that the pressure variations within the bifurcation zone (the size of which is of the order of the diameters) will be much smaller than the variations along the pipes. It leads to replace the actual 3 dimensional network of interconnected pipes by a one dimensional network, where the bifurcation zone has been reduced down to a bifurcation point, at which a single pressure $p$ is defined. Denoting by $u_{i}, i=0,1,2$ the fluxes through the pipes (considered positive whenever fluid flows out of the network), and $r_{i}, i=0,1,2$ the resistances estimated according to the approach detailed in the previous section, Poiseuille's law write

$$
p-p_{0}=r_{0} u_{0}, p-p_{1}=r_{1} u_{1}, p-p_{2}=r_{2} u_{2},
$$




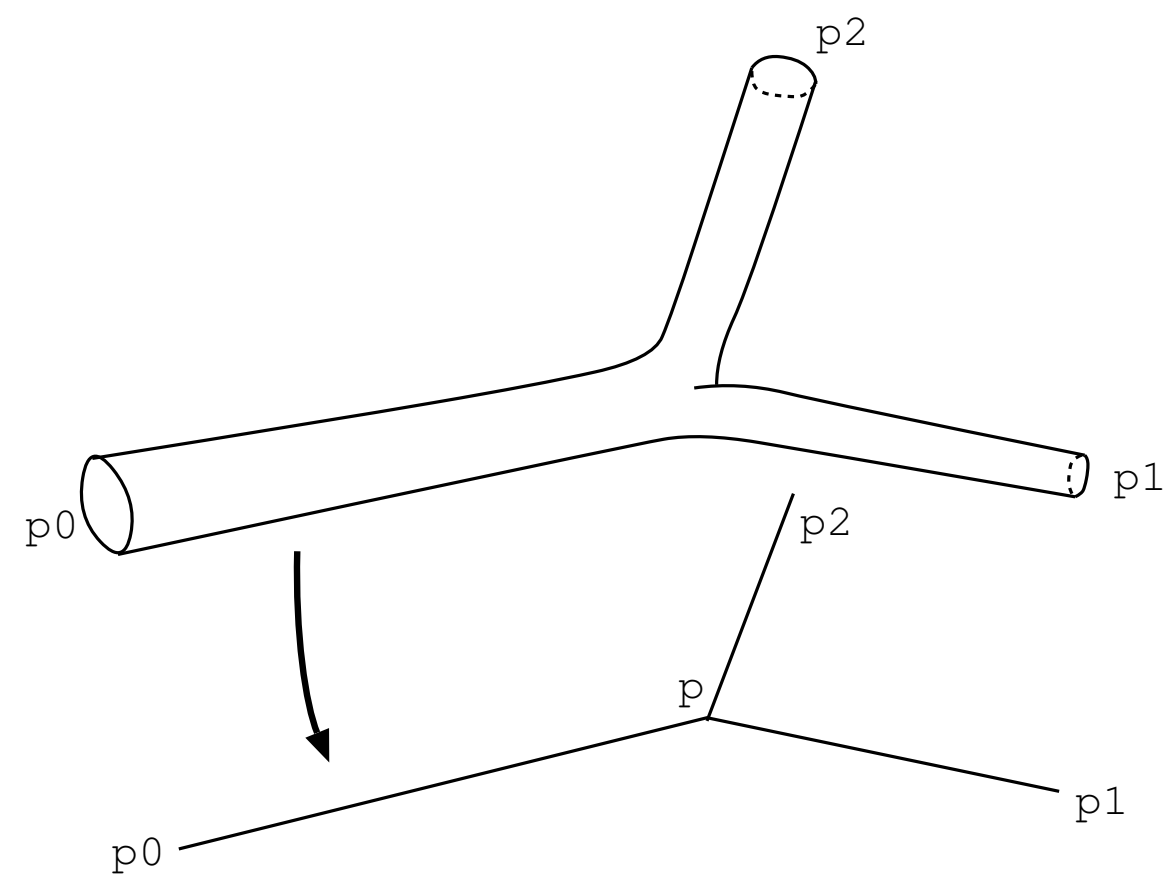

FiguRE 1. Stokes flow in a network

and the conservation of fluid (Kirchhoff's law) imposes

$$
u_{0}+u_{1}+u_{2}=0
$$

The symmetric tree model. The notion of resistance of a network will be detailed below, in a quite general setting (see Def. 2.3). Yet, in some cases, this resistance can be straightforwardly defined and computed. As detailed in [24], the respiratory tract, seen as a dyadic resistive tree, can be considered symmetric as a first approximation. It means in this context that all branches of a given generation have the same length and diameter. Making this approximation considerably simplifies the situation. Consider such a symmetric tree with $N$ generations, and denote by $r_{n}$ the resistance of any branch at generation $n$ (between 0 and $N$ ). We apply a unit pressure drop between the root and the boundary, that is the set of leafs at generation $N$ (there are $2^{N}$ of such leaves). By symmetry of the problem, all pressures will be the same at all vertices of a given generation. As a consequence, the $n$-th generation can be seen as a single resistance $r_{n} / 2^{n}$ (resistances in parallel). The generations being in series, the global resistance can be computed as

$$
R_{N}=\sum_{n=0}^{N} \frac{r_{n}}{2^{n}}, r_{n}=\frac{128 \mu}{\pi} \frac{\ell_{n}}{d_{n}^{4}},
$$

where $\ell_{n}$ and $d_{n}$ are the length and diameter associated to generation $n$. Typical values can be found in the literature, see Table 1, that is taken from [23]. We truncated the table after generation 16, since the contribution of further branches to the global resistance is negligible. The computation gives $R_{15}=0.28 \mathrm{~cm} \mathrm{H}_{2} \mathrm{O} \mathrm{s} \mathrm{L}^{-1}$. Explaining the gap between this value and the measured value (between 1 and $2 \mathrm{~cm} \mathrm{H}_{2} \mathrm{O} \mathrm{s} \mathrm{L}^{-1}$ ) goes far beyond the scope of this paper. Let us simply mention some resistance factors that have been disregarded here: inertial effects [18], upper airway resistance [26,27], resistance of the tissues [3] (although this part, strictly speaking, is not accounted for by the interrupter method mentioned above). We will nevertheless see in Section 3.2 that a 
significant part of this gap can be explained by the sole intrinsinc variability of geometrical parameters (branch dimensions).

\subsection{Abstract resistive networks}

The considerations pertaining to a single bifurcation can be extended to general networks of pipes. Let us present here a general framework to define the notion of global resistance. We refer to [2, 4, 7, 17, 21, 28] for thorough presentations of the underlying abstract notions, and to [5,11] for extensions of this framework to time-dependent (wave and heat) equations.

Definition 2.2. (Network, rooted network)

A finite resistive network is a triplet $N=(V, E, r)$, where $V$ is a finite set of vertices, $E \subset V \times V$ is the set of edges, symmetric $((x, y) \in E \Longrightarrow(y, x) \in E)$, and $r$ is the resistance field defined in $E(r(x, y)=r(y, x)$ for any $(x, y) \in E)$. Resistances are assumed to be positive. We shall say that a connected network $N=(V, E, r)$ is a rooted network when a vertex $o$ has been singled out as the root, together with a non empty subset $\Gamma$ of

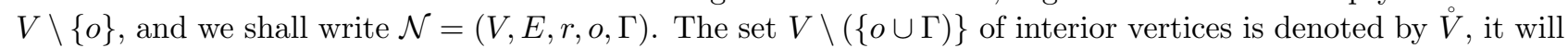
correspond to vertices that are subject to mass balance, whereas some fluid can be exchanged with the outside world though vertices in $\Gamma$, or through the root $o$.

One considers a pressure field as a collection of real values at vertices $\left(p \in \mathbb{R}^{V}\right)$, and flux fields as a collection of values on edges $\left(u \in \mathbb{R}^{E}\right)$. Fluxes are skew-symmetric: $u(x, y)=-u(y, x)$.

For any edge $e=(x, y)$ of the network, Poiseuille's law writes

$$
p(x)-p(y)=r(x, y) u(x, y)=r(e) u(e)
$$

Now if one denotes by $j(x)$ the flow rate injected in the network at $x$, Kirchhof's law writes,

$$
\sum_{y \sim x} u(x, y)=j(x),
$$

where $y \sim x$ means that $y$ is connected to $x$ (i.e. $(x, y) \in E$ ). Since we assume mass balance at interior vertices, we have $j(x)=0$ for $x \in \stackrel{\circ}{V}$.

We shall denote by $d$ the discrete divergence operator (it is actually the opposite of the divergence operator)

$$
\begin{aligned}
d: u \in \mathbb{R}^{E} & \longmapsto d u \in \mathbb{R}^{V} \\
d u(x) & =-\sum_{y \sim x} u(x, y) .
\end{aligned}
$$

In what follows we shall be interested in conservative fluxes, i.e. fluxes $u$ such that $d u(x)=0$ for any vertex $x$ in $\stackrel{\circ}{V}=V \backslash(\{o\} \cup \Gamma)$. We define its formal adjoint $d^{\star}$ (discrete counterpart of the gradient operator) as

$$
\begin{aligned}
d: p \in \mathbb{R}^{V} & \longmapsto d^{\star} p \in \mathbb{R}^{E} \\
d^{\star} p(e) & =p(y)-p(x) .
\end{aligned}
$$

Writing Poiseuille's and Kirchhoff's laws leads to a Darcy-like problem

$$
\left\{\begin{array}{llll}
u+c d^{\star} p & = & 0 & \text { in } E \\
d u & = & 0 & \text { in } \stackrel{\circ}{V} .
\end{array}\right.
$$

where $c$ (conductance field) stands for $1 / r$, i.e. $c(e)=1 / r(e)$ for any $e \in E$. Consider now the problem that consists in computing pressures and fluxes overall the network, when pressure values are prescribed at $o$ and on 
$\Gamma$ (the network is assumed to be rooted). By eliminating the velocity, this problem writes as a discrete Poisson problem for the pressure, with Dirichlet boundary conditions:

$$
\left\{\begin{aligned}
d c d^{\star} p(x) & =0 \quad \forall x \in \stackrel{\circ}{V}, \\
p(o) & =0 \\
p(x) & =P(x) \quad \forall x \in \Gamma,
\end{aligned}\right.
$$

where $P$ is a collection of prescribed pressures over the boundary $\Gamma$. Well-posedness of this problem is straightforward, as soon as the network is connected. Indeed, the bilinear form

$$
(p, q) \longmapsto a(p, q)=\sum_{e} c(e)(p(y)-p(x))(q(y)-q(x)),
$$

is coercive, so that Lax-Milgram theorem applies. Note that

$$
a(p, p)=\sum_{e} c(e)|p(y)-p(x)|^{2}
$$

is the rate of energy dissipation within the network. The definition of the network resistance follows.

Definition 2.3. (Effective resistance of a network)

Let $\mathcal{N}=(V, E, r, o, \Gamma)$ be a rooted network according to Def. 2.2. We consider a uniform pressure field $P \equiv 1$ on $\Gamma$. We denote by $p$ the solution to Dirichlet problem (11), and by $u=-c d^{\star} p$ the associated flux field. The global flux $Q$ is obtained by summing up fluxes flowing in the network through $\Gamma$, or equivalently flowing out through $o$ :

$$
Q=-\sum_{x \sim o} u(o, x)=d u(o)
$$

The equivalent resistance of $\mathcal{N}$ is defined as $R(\mathcal{N})=1 / Q$. By linearity, the flux associated to a non unit uniforme pressure $P$ on $\Gamma$ verifies $P-0=R Q$.

The definition finds support in the energy balance, which can be written like in the Darcy setting.

Proposition 2.4. (Joule's law for a network)

Let $\mathcal{N}=(V, E, r, o, \Gamma)$ be a rooted network, and $p$ the solution to Problem (11) with a uniform pressure P. The rate of dissipated energy within the network is

$$
\mathcal{P}=R Q^{2}
$$

where $Q=d u(o)$ is the flux from $\Gamma$ to $o$.

Proof. This is a direct consequence of the discrete counterpart of Green's formula (summation by parts). The dissipated energy writes

$$
\begin{aligned}
\mathcal{P} & =\sum_{E} c(x, y)(p(x)-p(y))^{2} \\
& =\sum_{x \in \dot{V}} p(x) \underbrace{\sum_{y \sim x} c(x, y)(p(x)-p(y))}_{=d c d^{\star} p(x)=0}+\sum_{x \in\{o\} \cup \Gamma} p(x) \sum_{y \sim x} c(x, y)(p(x)-p(y)) \\
& =P \sum_{x \in \Gamma} d c d^{\star} p(x)=-P \sum_{x \in \Gamma} d u(x)=P d u(o)=R d u(o)^{2}
\end{aligned}
$$

which ends the proof. 
Remark 2.5. Let us stress out similarities and differences between the discrete setting and the continuous one (Darcy equations (8)). The Green formula that we used in the previous proof

$$
\sum_{E} c(x, y)(p(x)-p(y))(q(x)-q(y))=\sum_{x \in V} q(x) \sum_{y \sim x} c(x, y)(p(x)-p(y))
$$

is similar to the standard Green formula in a euclidean domain with no boundary (e.g. for periodic, or infinite domains). Indeed, the notion of "boundary" in a network is arbitrary, and we did not make any topological assumptions on the vertices that belong to $\Gamma$. In particular, they might have an arbitrary number of neighbors, i.e. they might be within the network. We obtained pseudo boundary terms by decomposing the vertex set into $\stackrel{\circ}{V}$ and $\{o\} \cup \Gamma$, and the corresponding formula does not really have a continuous counterpart. Indeed, in the continuous setting, it would consist in considering the Poisson problem

$$
-\Delta \mathrm{p}=0 \quad \text { in } \Omega \backslash X
$$

where $\Omega$ is a domain without boundary, and $X$ a finite collection $\left(x_{i}\right)$ of points in $\Omega$, with a prescribed value $\mathrm{p}_{i}$ at $x_{i}$, so that

$$
-\Delta \mathrm{p}=\sum_{i} u_{i} \delta_{x_{i}}
$$

where $u_{i}$ is the flux inward the domain at $x_{i}$. Then, formally, we have

$$
\int_{\Omega}|\nabla \mathrm{p}|^{2}=\sum_{i} u_{i} \mathrm{p}_{i}
$$

that would be the continuous counterpart of (13). The problem is that it does not make proper sense, since points have zero capacity as soon as the space dimension is greater than 1.

To obtain a Green formula with a boundary term at the discrete level (discrete counterpart of $\int_{\Gamma} \partial p / \partial n$ ), one has to consider the set of "boundary edges" $E^{\Gamma}$, i.e. the set of all those edges that share a point with $\Gamma$ (with the convention that $(x, y) \in E^{\Gamma}$ as soon as $x \in \Gamma$ ). In this setting, we have

$$
\begin{aligned}
\sum_{E} c(x, y)(p(x)-p(y))(q(x)-q(y)) & =\sum_{x \in \dot{V}} q(x) \underbrace{\sum_{y \sim x} c(x, y)(p(x)-p(y))}_{=d c d^{\star} p(x)}+\sum_{x \in\{o\} \cup \Gamma} q(x) \sum_{y \sim x} c(x, y)(p(x)-p(y)) \\
& =\sum_{x \in \dot{V}} q(x) d c d^{\star} p(x)-\sum_{e=(x, y) \in E^{\Gamma}} c(x, y) q(x) d^{\star} p(e),
\end{aligned}
$$

that is now the proper counterpart of

$$
\int_{\Omega} k \nabla \mathrm{p} \cdot \nabla \mathrm{q}=-\int_{\Omega} q \nabla \cdot k \nabla \mathrm{p}+\int_{\Gamma} k \frac{\partial \mathrm{p}}{\partial n} .
$$

To end this remark, let us also mention that the discrete counter part of the Divergence theorem in a bounded domain

$$
\int_{\Omega} \nabla \cdot \mathbf{v}=\int_{\partial \Omega} \mathbf{v} \cdot \mathbf{n}
$$

is simply obtained by summing up the $d u(x)^{\prime} s$ over all the vertices (including those on the "boundary"). In the situation that we considered here, with $d u=0$ at interior points, we straightforwardly obtain

$$
d u(o)+\sum_{x \in \Gamma} d u(x)=0
$$


which simply expresses the global mass balance.

Resistance operator. The resistance of a network (between two subsets $\{o\}$ and $\Gamma$ ) has been defined as a real number that relates a uniform pressure applied on $\Gamma$ and the global flux (see Def. 2.3). When the applied pressure field is not uniform, it calls for a more general notion.

Definition 2.6. Let $\mathcal{N}=(V, E, r, o, \Gamma)$ be a rooted network, and let $\phi$ be a collection of fluxes entering the networks through $\Gamma$. We consider it as a function in $\mathbb{R}^{V}$, with $\phi(x)=0$ as soon as $x \notin \Gamma$, whenever needed. We define $p \in \mathbb{R}^{V}$ as the solution to

$$
\left\{\begin{aligned}
d c d^{\star} p(x) & =\phi(x) \quad \forall x \in \stackrel{\circ}{V} \cup \Gamma, \\
p(o) & =0
\end{aligned}\right.
$$

We denote by $p_{\Gamma}$ the collection of pressure values on $\Gamma$. The resistance operator is defined as

$$
\mathcal{R}: \phi \in \mathbb{R}^{\Gamma} \longmapsto p_{\Gamma} \in \mathbb{R}^{\Gamma},
$$

so that the generalized Poiseuille's law

holds.

$$
p_{\Gamma}=\mathcal{R} \phi
$$

The term $p_{\Gamma}=p_{\Gamma}-0$ above can be seen as a generalized pressure drop between $\Gamma$ and $o$, whereas $\phi$ is a generalized (i.e. vector) flux.

Remark 2.7. Note that, in the Darcy setting, the resistance operator can be defined as well, and it takes the form of a Neuman-Dirichlet operator. From this standpoint, one would expect a Neuman problem to be involved in the definition, whereas (15) is a Dirichlet problem. This apparent mismatch between the continuous and the discrete settings is again due to the fact that, in the discrete setting, Neuman conditions are actually handled as a non homogeneous right-hand side (see Remark 2.5 regarding this matter).

\subsection{Equivalent resistance for a dyadic tree}

In the case of a dyadic tree like the respiratory tract, the considerations above take a particular form. We consider here a finite, $N$-generation dyadic tree, with root $o$, which we suppose is set to pressure 0 . We denote by $\left(x_{n}^{k}\right)$ its vertices, and by $\left(e_{n}^{k}\right)$ its edges ${ }^{1}$, with $0 \leq n \leq N, 0 \leq k<2^{n}$. We denote by $p_{n}^{k}$ the pressure at node $x_{n}^{k}$, by $r_{n}^{k}$ the resistance of $e_{n}^{k}$ and by $u_{n}^{k}$ the flux through $e_{n}^{k}$ (see Fig. 2).

Poiseuille's law writes

$$
p_{n}^{k}-p_{n+1}^{2 k}=r_{n+1}^{2 k} u_{n+1}^{2 k}, p_{n}^{k}-p_{n+1}^{2 k+1}=r_{n+1}^{2 k+1} u_{n+1}^{2 k+1} \quad 0 \leq k<2^{n},
$$

and Kirchhoff's law

$$
u_{n}^{k}-u_{n+1}^{2 k}-u_{n+1}^{2 k+1}=0 .
$$

External pressure (at root $o$ ) being set to 0, the generalized Poiseuille's law across the tree takes the form of a linear relation between pressures $p=\left(p_{N}^{k}\right)_{0 \leq k<2^{N}}$ and fluxes $u=\left(u_{N}^{k}\right)_{0 \leq k<2^{N}}$

$$
0-p=R u
$$

and we aim at expressing how matrix $R$ depends on the resistances.

\footnotetext{
${ }^{1}$ Edges are chosen oriented from root to leafs, i.e.

$$
e_{n+1}^{2 k}=\left[x_{n}^{k}, x_{n+1}^{2 k}\right], e_{n+1}^{2 k+1}=\left[x_{n}^{k}, x_{n+1}^{2 k+1}\right] .
$$
}



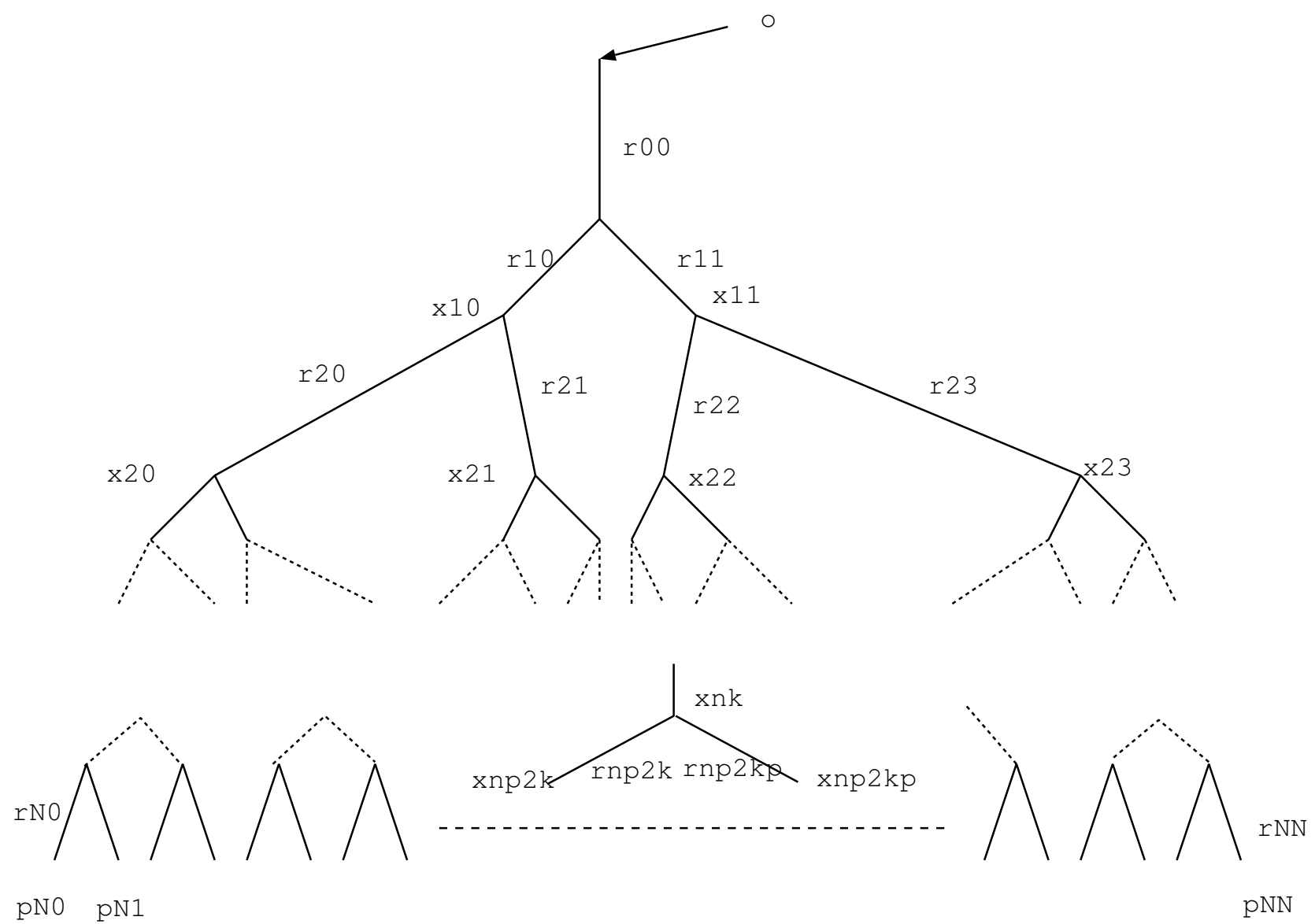

Figure 2. $N$-generation resistive tree

Resistance operator. Assume that end fluxes $u_{N}^{k}, 0 \leq k<2^{N}$ are known. Let us determine pressures along the path from 0 to $x_{N}^{0}$. Poiseuille's law for the first edge (connecting $o$ and $x_{0}^{0}$ ) writes

$$
0-p_{0}^{0}=r_{0}^{0} u_{0}^{0}
$$

with

$$
u_{0}^{0}=\sum_{k=0}^{2^{N}-1} u_{N}^{k}
$$

by mass conservation. At the next step

$$
p_{0}^{0}-p_{1}^{0}=r_{1}^{0} u_{1}^{0},
$$

where again $u_{1}^{0}$ can be computed as the sum of end fluxes over the first half of indices. We obtain recursively $p_{2}^{0}, p_{3}^{0}, \ldots$, and finally

$$
\begin{aligned}
p_{N}^{0} & =-r_{0}^{0} u_{0}^{0}-r_{1}^{0} u_{1}^{0}-\cdots-r_{N}^{0} u_{N}^{0} \\
& =-\sum_{n=0}^{N} r_{n}^{0}\left(\sum_{k=0}^{2^{(N-n)}-1} u_{N}^{k}\right) .
\end{aligned}
$$


This approach can be generalized to a path connecting $o$ to any endpoint $x_{N}^{k}$ :

$$
\left[o, x_{0}^{0}, x_{1}^{k_{1}}, \ldots, x_{n}^{k_{n}}, \ldots, x_{N}^{k}\right]
$$

which allows to express $p=\left(p_{N}^{k}\right)_{0 \leq k<2^{N}}$ with respect to fluxes as

$$
0-p=\mathcal{R} u
$$

where $\mathcal{R}$ is the resistance matrix, which is expressed below. Let $J_{n}$ be the $2^{n} \times 2^{n}$ matrix with all entries equal to 1 (one-rank matrix), $\mathcal{R}$ writes

$$
\begin{aligned}
\mathcal{R}=r_{0}^{0} J_{N}+\left(\begin{array}{cc}
r_{1}^{0} J_{N-1} & 0 \\
0 & r_{1}^{1} J_{N-1}
\end{array}\right)+\left(\begin{array}{ccccc}
r_{2}^{0} J_{N-2} & 0 & 0 & 0 \\
0 & r_{2}^{1} J_{N-2} & 0 & 0 \\
0 & 0 & r_{2}^{2} J_{N-2} & 0 \\
0 & & 0 & 0 & r_{2}^{3} J_{N-2}
\end{array}\right)+\ldots \\
+\left(\begin{array}{ccccc}
r_{N}^{0} & 0 & \ldots & \ldots & 0 \\
0 & r_{N}^{1} & 0 & \ldots & \vdots \\
\vdots & & \ddots & & \vdots \\
\vdots & & & \ddots & \vdots \\
0 & \ldots & \ldots & 0 & r_{N}^{2^{N}-1}
\end{array}\right)
\end{aligned}
$$

\subsection{The resistance operator as a convolution}

We introduced the resistance operator in a general setting at the end of Section 2.2, and in matrix form in Section 2.3 for a dyadic tree. We show here that, in an appropriate framework, this operator actually is of the convolution type, as soon as the considered tree is symmetric. We refer to [4] for a detailed description of this framework, and its extension to infinite trees.

Consider a $\mathrm{N}$-generation dyadic tree. Nodes of a given generation are indexed as shown in Fig. 3: starting from the root, a right turn is encoded by 0 , a left turn by 1 . A node at generation $N$ is represented by $N$ bits: $a_{N-1} a_{N-2} \ldots a_{0}$. We map this $N$-bit number onto $\mathbb{Z} / 2^{n} \mathbb{Z}$ in the following manner

$$
a_{N-1} a_{N-2} \ldots a_{0} \longmapsto \sum_{k=0}^{N-1} a_{k} 2^{k}
$$

considered as a element of $\mathbb{Z} / 2^{N} \mathbb{Z}$. This leads to an indexing of the boundary (set of leafs) $\Gamma_{N}$ of the $N$ generation 2 -adic tree, that is more natural, as we shall see, than the linear indexing $0,1,2, \ldots$ For instance, the indexing for $N=3$ is the following: $0,4,2,6,1,5,3,7$. Now any $a \in \mathbb{Z} / 2^{N} \mathbb{Z}$ can be written $a=a^{\prime} 2^{p}$, with $a^{\prime}$ odd. We define $p=p(a) \in[0, N-1]$ as the valuation of $a$. The 2-adic absolute value of $a$ is defined as $|a|_{2}=2^{-p(a)}$. The 2-adic distance between two vertices $a$ and $b$ of $\Gamma_{N}$ (identified to $\mathbb{Z} / 2^{N} \mathbb{Z}$ ) is then defined as $|b-a|_{2}$. This distance reflects the tree structure, in the sense that it is a monotone function of the graphdistance between vertices, defined as the length of the shortest path (in the tree) connecting two vertices of $\Gamma_{N}$. In particular, the distance is minimal $\left(1 / 2^{N-1}\right)$ when 2 leafs share a direct ascendant, and maximal (equal to 1) when they belong to different halves (in the case where the shortest path goes up to the top of the tree).

In this setting, the resistance operator defined by (16) takes on a particular form. In order to emphasize the convolution character of this operator, we shall use an integral formulation instead of a summation one. For a 


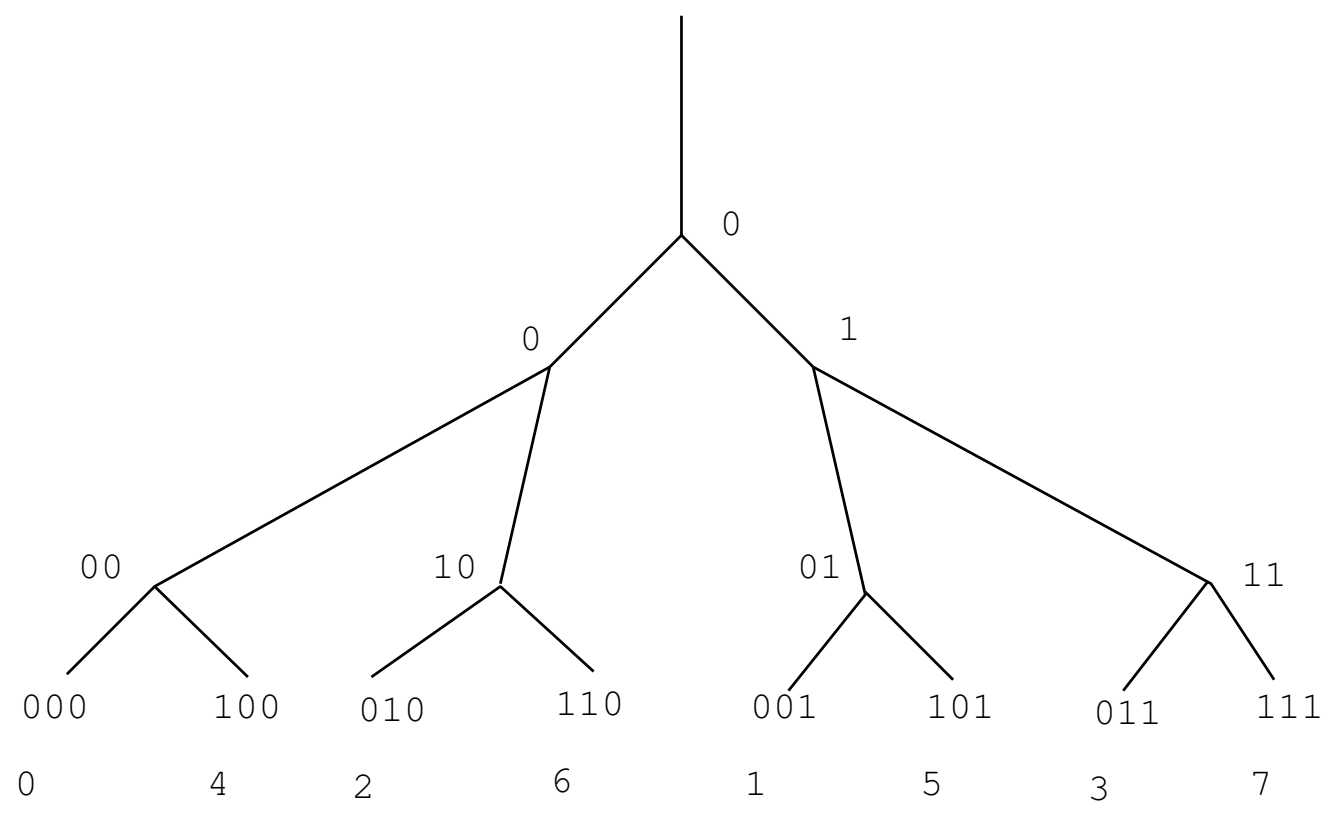

FiguRE 3. 2-adic indexing

function $g$ in $\mathbb{R}^{\Gamma_{N}}$ (that can be seen as a $2^{N}$ dimensional vector), we shall write

$$
\int_{\mathbb{Z} / 2^{N} \mathbb{Z}} g(x) d x=\frac{1}{2^{N}} \sum_{k=0}^{2^{N}-1} g(k),
$$

in the spirit of Haar's measure on $\mathbb{Z}_{2}$, the ring of 2-adic integers (see [4]). The underlying measure is such that a set of the form $z+2\left(\mathbb{Z} / 2^{N} \mathbb{Z}\right)$, has total measure $1 / 2$. Similarly, the set $z+2^{p}\left(\mathbb{Z} / 2^{N} \mathbb{Z}\right)$ has a measure $1 / 2^{p}$, for $p \leq N$, for any $z$.

Proposition 2.8. Consider a symmetric $N$-generation dyadic tree (i.e. the resistance is the same for all branches of the same generation). The resistance operator defined in a general setting by Def. 2.6, and for dyadic trees by the matrix (16), can be written

$$
p(x)=\mathcal{R} q(x)=\int_{\mathbb{Z} / 2^{n} \mathbb{Z}} \Psi(x-y) q(y) d y .
$$

Proof. This is a direct consequence of the matrix expression (16), in the case where the resistance does not depend on the branch within each generation, i.e. $r_{n}^{k} \equiv r_{n}$. Consider for instance a flux vector that is 1 at 0 , and 0 at all other vertices. For all those vertices that are not in the same half than 0 , the pressure is simply $r_{0}$. For all those that are in the same half, but not in the same quarter, the pressure is $r_{0}+r_{1}$, etc... In the 2 -adic setting it suffices to define $\Psi$ by

$$
\Psi: x \in \mathbb{Z} / 2^{N} \mathbb{Z} \longmapsto \Psi(x)=r_{0}+r_{1}+\cdots+r_{k}, \text { with } k=-\log _{2}\left(|x|_{2}\right),
$$

to obtain the expression (17). 


\subsection{Stochastic setting}

We briefly describe here the links between the discrete Laplace problem and a stochastic process. Further details about this setting can be found in [7]. This section is purely abstract in the sense that the random walk that we will describe is not related to any actual process in the physical space, but it enlightens the mathematical structure of the problem, and it can be used to compute equivalent resistances by a Monte Carlo algorithm.

Considering a Network $\mathcal{N}=(V, E, r)$, we define a random walk by the transition probabilities $\pi_{x y}$, where $x$ and $y$ range over the set of vertices. This transition probability is defined as

$$
\pi_{x y}=\frac{c(x, y)}{C(x)}, C(x)=\sum_{y \sim x} c(x, y)
$$

where $c(x, y)=1 / r(x, y)$ is the conductance of edge $(x, y)$. The probability to go from $x$ to $y$ is 0 as soon as $y$ is not connected to $x$. The corresponding Markov chain is irreducible as soon as the network is connected, which we assume here.

Now consider a rooted network $\mathcal{N}=(V, E, r, o, \Gamma)$ and Dirichlet data on $\Gamma$ : a collection of values $(P(x))_{x \in \Gamma}$ is prescribed. We define $p \in \mathbb{R}^{V}$ as follows: considering a vertex $x \in V$, we denote by $i$ the step that corresponds to the first hitting with $\Gamma$ or $o$ of the random walk starting from $x$ :

$$
X_{0}=x, X_{1}, \ldots, X_{i} \in \Gamma \cup\{o\}
$$

with $X_{j} \notin \Gamma \cup\{o\}$ for $0<j<i$. The value of $P$ at $X_{i}$ (it is zero whenever $X_{i}=o$ ) is a random variable. We denote by $p(x)$ the corresponding expected value. We have the following link between this process and Dirichlet Problem (11).

Proposition 2.9. Let $p \in \mathbb{R}^{V}$ be defined as previously. Then $p$ is the solution to Problem (11).

Proof. Let us first notice that Dirichlet boundary conditions are automatically verified (in the case $x \in \Gamma \cup\{o\}$, the first hitting index $i$ is 0$)$. Now consider $x \in \stackrel{\circ}{V}$. It holds

$$
p(x)=\sum_{y \sim x} \pi_{x y} p(y)
$$

which can be written (from (18))

$$
C(x) p(x)-\sum_{y \sim x} c(x, y) p(y)=0,
$$

so that $p$ is harmonic. As a consequence, it is the unique solution to (11).

This property can be used to obtain a stochastic expression of the resistance between $o$ and $\Gamma$. Consider the case where $P \equiv 1$. The field $p$ defined previously is then the escape probability: for $x \in V$, it represents the probability that the random walk starting from $x$ hits $\Gamma$ (i.e. "escapes") before hitting $\Gamma$.

Proposition 2.10. Consider a random walk starting from o, transition probabilities given by (18). It holds

$$
\frac{1}{R}=C(o) p_{e s c}
$$

where $p_{\text {esc }}$ is the probability that the random walk hits $\Gamma$ before returning to o, and $R$ the resistance between o and $\Gamma$ (see Def. 2.3). 
Proof. Let $p$ be the solution to the Dirichlet problem (11). By Definition 2.3, the resistance $R$ is $1 / d(o)$. Now by Prop. 2.9, the escape probability is

$$
p_{e s c}=\sum_{x \sim o} \pi_{o x} p(x)=\frac{1}{C(o)} \sum_{x \sim o} c(o, x)(p(x)-p(o))=\frac{1}{C(o)} d u(o)=\frac{1}{C(o)} \frac{1}{R},
$$

which yields the result.

\subsection{Actual computation of the equivalent resistance for a general dyadic tree}

The actual computation of the equivalent resistance of a network is dictated by Def. 2.3. It amounts to solving the discrete Dirichlet problem (11), with $P \equiv 1$, and then compute the resistance as $R=1 / d u(o)$. In the case of a $N$-generation dyadic tree, an alternative approach can be undertaken, by means of the resistance operator $\mathcal{R}$, defined in matrix form by (16). Consider a unit pressure field $p=(1,1, \ldots, 1) \in \mathbb{R}^{2^{N}}$, solve

$$
\mathcal{R} j=p,
$$

and then compute the total flux as the sum of elements of $j$. The advantage is not obvious, since the straight approach consists in solving a $2^{N+1} \times 2^{N+1}$ system for a very sparse matrix (the maximal number of non-zero entries in a row is 4), whereas the Neuman-Dirichlet approach that we propose here consists in solving a slightly smaller linear system $\left(2^{N} \times 2^{N}\right)$, but with a full matrix $\mathcal{R}$. Yet, the particular structure of $\mathcal{R}$, (see (16)), makes it possible to design a fast matrix-vector product. We describe the idea of the method for the linear indexing (that corresponds to the matrix expression (16)), but it could be also be formulated within the 2-adic setting presented in Section 2.4. For a given flux field $q$, the matrix-vector product $\mathcal{R}$ requires the computations of partial sums

and then

$$
q_{0}+q_{1}, q_{2}+q_{3}, q_{4}+q_{5}, \ldots
$$

$$
q_{0}+q_{1}+q_{2}+q_{3}, q_{4}+q_{5}+q_{6}+q_{7}, \ldots \text { etc }
$$

Once the 2-term sums have been computed, it is clear that each 4-term sum only requires a single addition (and not 3). Recursively computing those sums to perform the matrix-vector product leads to a very efficient algorithm. The linear system can then be solved by an iterative algorithm, like the conjugate gradient algorithm. Note that a Monte Carlo method can also be undertaken in the spirit of Section 2.5, according to Formula (19). This approach is less accurate than the direct one (described previously), but it can be useful in the case where some individual resistances are very large. Indeed, the large condition number of the matrix is likely to harm the convergence speed of any iterative method, while it will not affect the behavior of the Monte Carlo strategy. We refer to [16] for a detailed comparison of both approaches, in various situations.

\section{Optimality And stability issues}

This section addresses natural questions concerning optimality and robustness of the lung as a resistive tree, both from the standpoint of constrained optimization and from the statistical standpoint.

\subsection{Minimizing the cost of breathing}

There is an increasing number of research papers dedicated to optimality of physiological systems. We must stress out that the answer to the question:

$$
\text { Is the system optimal? }
$$

strongly depends upon the meaning that is given to optimality, and upon the constraints that are accounted for in setting optimization problems. Having said that, it is to be expected that the evolutionary process has favored shapes and structures that provide some efficiency to the overall system. As for the ventilation process, if we consider as given that it aims at renewing alveolar air to improve passive diffusion by maintaining a 
required tidal volume (see Section 1), an obvious criterium is the energy cost. A first attempt in this direction was proposed in [13].

The authors consider a $N$-generation dyadic tree that is assumed to be symmetric. They furthermore assume that all branches over the tree have the same aspect ratio: if $\ell_{n}$ is the length at generation $n$, the diameter is $c \ell_{n}$, so that the individual resistance of a branch linearly depends on $1 / \ell_{n}^{3}$, see Eq. (9). To alleviate notations, we shall consider that the resistance of a pipe at generation $n$ is simply $r_{n}=1 / \ell_{n}^{3}$ (we drop the multiplicative constant). Similarly, each branch at generation $n$ occupies a volume $\ell_{n}^{3}$. For a unit flow rate, the dissipated power reduces to the global resistance (see Prop. 2.4), that is

$$
\mathcal{P}=\mathcal{R}=\sum_{n=0}^{N} \frac{1}{2^{n} \ell_{n}^{3}} .
$$

whereas the total volume is

$$
V=\sum_{n=0}^{N} 2^{n} \ell_{n}^{3} .
$$

Considering that the volume is subject to remain below a maximal value, minimization of the resistance under this constraint can be solved exactly.

Proposition 3.1. (From [13])

Under the previous assumptions, minimality of $R$ under the maximal volume constraint is achieved for a geometric progression of the sizes, i.e.

$$
\ell_{n}=\beta \lambda^{n}, \text { with } \lambda=2^{-1 / 3},
$$

where $\beta$ is a normalization constant which ensures that the volume constrained is saturated.

Proof. This is a straightforward application of the Kuhn-Tucker necessary conditions for optimality under constraint.

This academic result is quite striking, since it can be assessed from actual measurements (see [24], or Table 1 taken from [23]) that the progression is indeed not far from being geometric, at least in the central range of generations (i.e. from 3 to 16 ). Furthermore, the measured value, that is 0.85 , is quite close to the "ideal" one $2^{-1 / 3} \approx 0.79$. We refer the reader to [13] for further discussions on the gap between the two values.

Note that the constrained optimization can be set in a different manner, by disregarding the maximal volume constraint, and replacing it by a constraint based on lengths. Assuming that the entrance of the respiratory system is necessary at a prescribed distance of the zone it is meant to irrigate, we may consider that the length of any path from the root to the leaf is fixed, i.e.

$$
\ell_{\text {tot }}=\sum_{n=0}^{N} \ell_{n}
$$

is prescribed. It can be shown with similar arguments (see [16]) that the optimum still exhibits a geometric character, with a ratio $1 / \sqrt[4]{2} \approx 0.84$ that is even closer to the experimental one.

We shall end this section by a very simple and general property of resistive networks, that concerns the control of the flux by external pressures. As for the lung, the question can be formulated as: would it be significantly more efficient to have a better control of the pressure field at the leafs of the trees (i.e. at the level of alveoli)? As we know, the basic control of the ventilation is quite poor, it relies on a single action, that is the contraction of the diaphragm. In a more academic way, it can be formulated as follows: Consider a rooted network $\mathcal{N}=(V, E, r, o, \Gamma)$ (see Def. 2.2) that carries a unit flux through its root, what is the pressure field on $\Gamma$ that ensures minimalty of the dissipated energy. As stated in the following proposition, the corresponding field is uniform. 
Proposition 3.2. Among all those pressure fields on $V$ that vanish at o, harmonic over the set of interior vertices $\stackrel{\circ}{V}=V \backslash(\{o\} \cup \Gamma)$, and driving a unit flux through o (or, equivalently, the opposite flux through $\Gamma$ ), the one that minimizes the dissipated energy is uniform over $\Gamma$.

Proof. Let us denote by $H$ the set of pressure fields that vanish at 0 . The problem consists in minimizing

$$
\frac{1}{2} \sum_{e \in E} c(x, y)(p(x)-p(y))^{2}
$$

over

$$
\left\{q \in H_{0}^{1}, d c d^{\star} q(o)=1\right\}, \text { with } H_{0}^{1}=\left\{q \in \mathbb{R}^{V}, q(o)=0 .\right\}
$$

By global conservation (see (14)), the flux constraint can be written (with $u=-c d^{\star} q$ )

$$
1=d c d^{\star} q(o)=-d u(o)=+\sum_{x \in \Gamma} d u(x)=-\sum_{x \in \Gamma} d c d^{\star} p(x) .
$$

The problem can be set as a saddle point-problem (see e.g. [6]): it amounts to find a couple $(p, \lambda) \in H_{0}^{1}$ that is a saddle-point for the Lagrangian

$$
\mathcal{L}(q, \mu)=\frac{1}{2} \sum_{e \in E} c(x, y)(q(x)-q(y))^{2}+\lambda\left(\sum_{x \in \Gamma} d c d^{\star} q(x)+1\right)
$$

The optimality conditions for the primal component write in a variational way as

$$
\sum_{e \in E} c(x, y)(p(x)-p(y))(q(x)-q(y))+\lambda \sum_{x \in \Gamma} d c d^{\star} q(x)=0 \quad \forall q \in H_{0}^{1}, \text { harmonic on } \stackrel{\circ}{V} \text {. }
$$

Applying properly the discrete Green formula (13) to the first term, we obtain

$$
\sum_{x \in \hat{V}} p(x) d c d^{\star} q(x)+\sum_{x \in \Gamma} p(x) d c d^{\star} q(x)+\lambda \sum_{x \in \Gamma} d c d^{\star} q(x)=0 \quad \forall q \in H_{0}^{1} .
$$

Now for any collection of fluxes $j \in \mathbb{R}^{\Gamma}$ (extended by 0 for vertices in $\stackrel{\circ}{V}$ ), we may consider the solution $q \in H_{0}^{1}$ to

$$
d c d^{\star} q(x)=j(x) \quad \forall x \in \stackrel{\circ}{V} \cup \Gamma .
$$

Plugging this very field in the variational formulation above leads to

$$
\sum_{x \in \Gamma}(p(x)+\lambda) j(x)=0 .
$$

Since $j$ can be chosen arbitrarily, it implies that $p \equiv-\lambda$ on $\Gamma$, hence the pressure field is uniform.

\subsection{Resistance dispersion and generation-wise Sobol sensitivity indices}

We consider the respiratory tract as an $N$-generation dyadic tree. According to the previous considerations, the global resistance $R$ depends (in a highly complex way) upon geometric characteristics (lengths and diameters) of all branches. Morphometric data can be found in the literature (see e.g. [23, 24]). Table 1 is taken from [23], it gives the mean lengths and diameters for the typical respiratory tract of an adult subject. 
TABLE 1. Lengths and diameters, with relative variations (from [23])

\begin{tabular}{|c||c|c||c|c||}
\hline Generation & Length $(\mathrm{mm})$ & $\sigma / m$ & Diam. $(\mathrm{mm})$ & $\sigma / m$ \\
\hline 0 & 105 & 0.1 & 15.7 & 0.1 \\
\hline 1 & 41.6 & 0.15 & 10.1 & 0.1 \\
\hline 2 & 16.6 & 0.25 & 7.3 & 0.125 \\
\hline 3 & 6.6 & 0.3 & 4.9 & 0.175 \\
\hline 4 & 11.1 & 0.35 & 3.9 & 0.2 \\
\hline 5 & 9.4 & 0.425 & 3.1 & 0.23 \\
\hline 6 & 7.9 & 0.5 & 2.5 & 0.275 \\
\hline 7 & 6.7 & 0.575 & 2.0 & 0.325 \\
\hline 8 & 5.6 & 0.65 & 1.6 & 0.35 \\
\hline 9 & 4.7 & 0.70 & 1.35 & 0.42 \\
\hline 10 & 4.0 & 0.75 & 1.14 & 0.5 \\
\hline 11 & 3.4 & 0.80 & 0.95 & 0.575 \\
\hline 12 & 2.9 & 0.81 & 0.83 & 0.66 \\
\hline 13 & 2.4 & 0.775 & 0.72 & 0.675 \\
\hline 14 & 2.0 & 0.725 & 0.65 & 0.6 \\
\hline 15 & 1.7 & 0.65 & 0.58 & 0.5 \\
\hline
\end{tabular}

Beside mean values, the table indicates coefficients of variation. Those are defined as the dimensionless ratio of standard deviation and mean value. They progress along the tree from small values (about 10\%) to much higher values (about $80 \%$ for generations 11 and 12). The variability that can be induced for the resistance of individual branches is huge. Consider for exemple a branch at generation 10, assume that its diameter and its length are (independent) random variables that follow a log-normal law, with mean values and standard deviations given by Table 1 . The corresponding resistance can be considered as a random variable. The mean and the standard deviation can be estimated as

$$
r_{10}=222 \mathrm{~cm} \mathrm{H}_{2} \mathrm{O} \mathrm{s} \mathrm{L}^{-1}, \sigma_{10} \approx 1400 \mathrm{~cm} \mathrm{H}_{2} \mathrm{O} \mathrm{s} \mathrm{L}^{-1} .
$$

Note that the mean value is more than 100 times the resistance of the overall tree. Furthermore, the coefficient of variation of the individual resistance is about $600 \%$. We aim here at investigating the real effect of this huge individual variability upon the global resistance.

We denote by $X_{n}$ the vector of lengths and diameters of the $2^{n}$ branches of generation $n$ :

$$
X_{n}=\left(\ell_{n}^{0}, \ell_{n}^{1}, \ldots, \ell_{n}^{2^{n}-1}, d_{n}^{0}, d_{n}^{1}, \ldots, d_{n}^{2^{n}-1}\right) .
$$

The global resistance $R$ can be written as a function of the geometric characteristics (lengths and diameters) of all branches, i.e.

$$
R=R\left(X_{0}, X_{1}, X_{2}, \ldots, X_{N}\right) .
$$

Following [23], we now assume that all diameters and lengths are independent random variables, so that $R$ is itself a random variable. Table 1 gives experimental values for the lengths and diameters of the branches over the generation range, together with associated coefficients of variation (dimensionless number, defined as the standard deviation divided by the mean value). Following [23] again, we shall consider each geometric parameter follows a log-normal distribution. Let us consider one of those variables (length or diameter at some generation $n$ ), denoted by $X$, with mean $m$ and standard deviation $\sigma$. It consists in writing

$$
X=e^{Y}, Y \sim \mathcal{N}(\mu, \tau)
$$




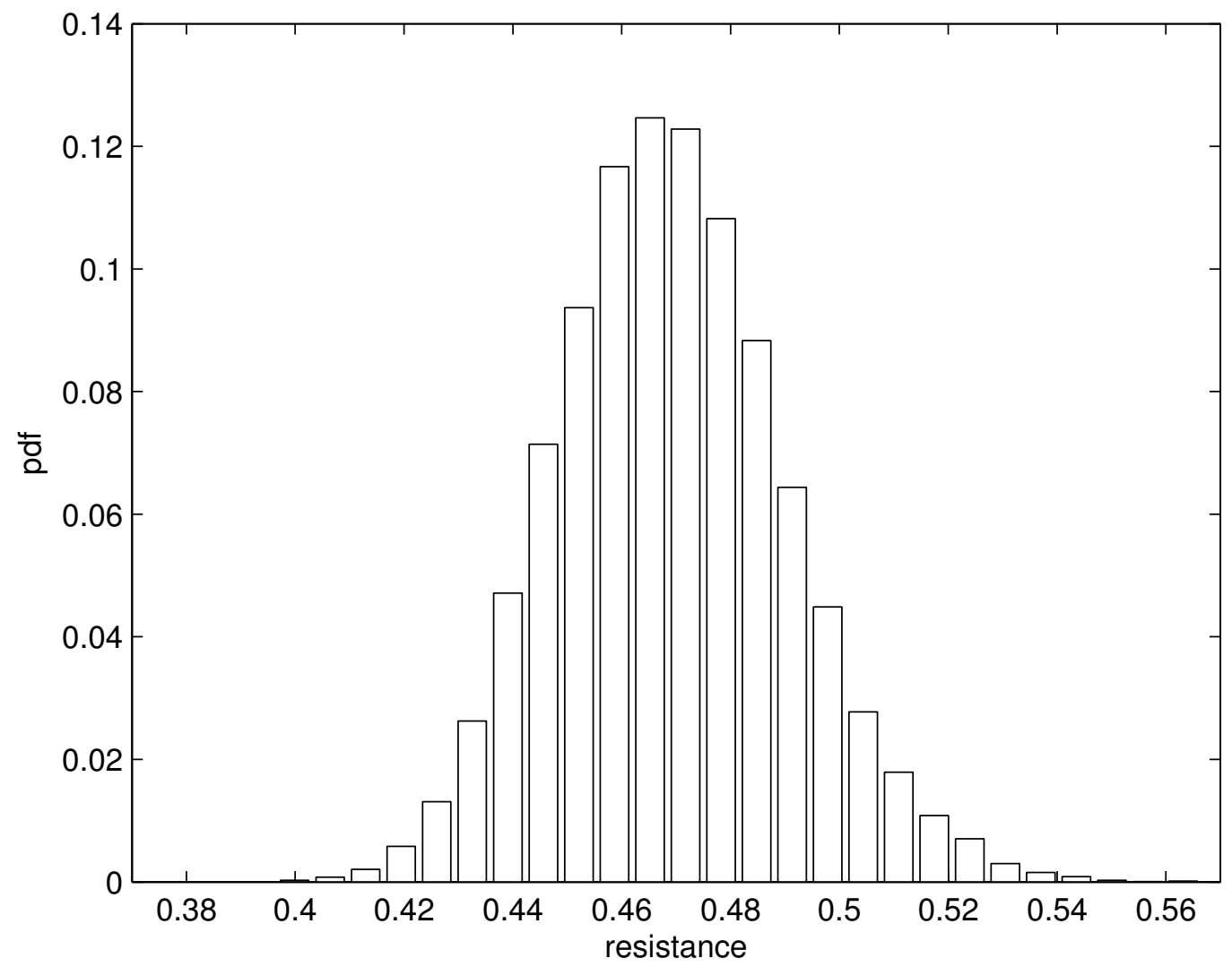

Figure 4. Resistance distribution

where $\mathcal{N}$ is a normal law with mean $\mu$ and standard deviation $\tau$, with

$$
\mu=\log \left(\frac{m}{\sqrt{1+\sigma^{2} / m^{2}}}\right), \tau=\sqrt{\log \left(1+\frac{\sigma^{2}}{m^{2}}\right)} .
$$

We shall therefore consider that all geometric quantities $\ell_{n}^{k}$ and $d_{n}^{k}$ follow such laws, the parameters of which only depends on generation index $n$, and that all random variables are independent.

Actual computation of equivalent resistances associated to randomly generated trees can be performed (see section 2.6), and Fig. 4 shows the obtained histogram (with 30000 samples). Two remarks are in order. First, the mean value $\left(0.47 \mathrm{~cm} \mathrm{H}_{2} \mathrm{O} \mathrm{s} \mathrm{L}^{-1}\right)$ is significantly higher that the value computed for the symmetric tree, with the mean values given by Table $1\left(0.28 \mathrm{~cm} \mathrm{H}_{2} \mathrm{O} \mathrm{s} \mathrm{L}^{-1}\right)$. Second, the distribution is narrow: the standard deviation is 0.02 , that is about $4 \%$ of the mean value. This asserts the strong robustness of the tree resistance with respect to the variability of constitutive parameters.

Remark 3.3. The previous computations are based on log-normal distributions for the lengths and diameters. Another natural choice in such a situation is the Gamma-distribution. In the present context, similar computations based on this alternative choice lead to a value of $R=0.51 \pm 0.03 \mathrm{~cm} \mathrm{H}_{2} \mathrm{O} \mathrm{s} \mathrm{L}^{-1}$. 
We now aim at quantifying the role played by the variability within each generation upon the global variability of the resistance, by means of Sobol sensitivity indices (see e.g. [22]). In this spirit, for any $n$, any $X_{n}$, we define $R_{n}\left(X_{n}\right)$ as

$$
R_{n}\left(X_{n}\right)=\mathbb{E}\left(R(X) \mid X_{n}\right)
$$

This quantity is a random variable that depends on $X_{n}$ only. We denote by $\sigma_{n}^{2}$ its variance. The $n-$ th Sobol index is then defined as

$$
S_{n}=\frac{\sigma_{n}^{2}}{\sigma^{2}}
$$

where $\sigma$ is the standard deviation of $R$. This dimensionless index $S_{n}$, between 0 and 1 , quantifies the part of the global variance that is explained by generation $n$.

Estimation of $S_{n}$. For any generation $n$, the index $S_{n}$ can be estimated by means of a Monte Carlo algorithm. It is based on generating samplings of the full vector of parameters

$$
X=\left(X_{0}, X_{1}, \ldots, X_{n-1}, X_{n}, X_{n+1}, \ldots, X_{N}\right)
$$

together with samplings of

$$
X^{\prime}=\left(X_{0}^{\prime}, X_{1}^{\prime}, \ldots, X_{n-1}^{\prime}, X_{n}, X_{n+1}^{\prime}, \ldots, X_{N}^{\prime}\right)
$$

It holds

$$
S_{n}=\frac{\operatorname{cov}\left(R, R^{\prime}\right)}{\operatorname{var}(\mathrm{R})}, \text { with } R=R(X), R^{\prime}=R\left(X^{\prime}\right) .
$$

Let $R_{1}, R_{2}, \ldots, R_{1}^{\prime}, R_{2}^{\prime}, \ldots$ be the sampled values. The Monte Carlo approach is then based on the following estimator (see [10]):

$$
\hat{S}_{n}=\frac{\frac{1}{K} \sum_{k=1}^{K} R_{k} R_{k}^{\prime}-\left(\frac{1}{K} \sum_{k=1}^{K} R_{k}\right)\left(\frac{1}{K} \sum_{k=1}^{K} R_{k}^{\prime}\right)}{\frac{1}{K} \sum_{k=1}^{K}\left(R_{k}\right)^{2}-\left(\frac{1}{K} \sum_{k=1}^{K} R_{k}\right)^{2}} .
$$

The results are presented in Fig. 5, for $K=10000$ samples for each generation. The standard deviations for each index (represented in the figure) is estimated by bootstrapping (random sampling with replacement from the same sample set of values for $R, R^{\prime}$ ). It appears that the largest part of the variability is due to the proximal generations (generations 0 to 8 ), whereas further (distal) generations play essentially no role in this variability. Another striking fact is the balanced distribution of indices in the proximal part: they vary between 0.05 and 0.15 . It suggests that evolutionary principles might tend to equi-distribute variability contributions in some complex systems like the respiratory tract.

\section{Conclusion}

Starting from the Top-Down (or functionalist) notion of resistance as a single scalar that can be measured from the outside, we described here frameworks that have been elaborated to properly define such a notion at a mathematical level. This approach makes it possible to handle the whole geometric complexity of the respiratory tract. We acknowledge the apparent futility in the process: considering a living subject, a full and accurate knowledge of geometric characteristics of his/her respiratory tract is clearly out of reach. Yet, computing equivalent resistances for general (i.e. non-symmetric) trees makes it possible to investigate the effect of microscopic variability (at the level of individual branches) over the global resistance. The main outcomes of these direct computations are

(1) The variability of individual branch characteristics has a significant effect upon the mean value of the effective resistance. As we pointed out in Section 3.2, the effective mean value is about twice the resistance computed from the mean geometric parameters.

(2) On the other hand, the high variability of local parameters does not induce a wide dispersion of the resistance values. The effective resistance distribution is very narrow (mean value $\pm 5 \%$ ). 


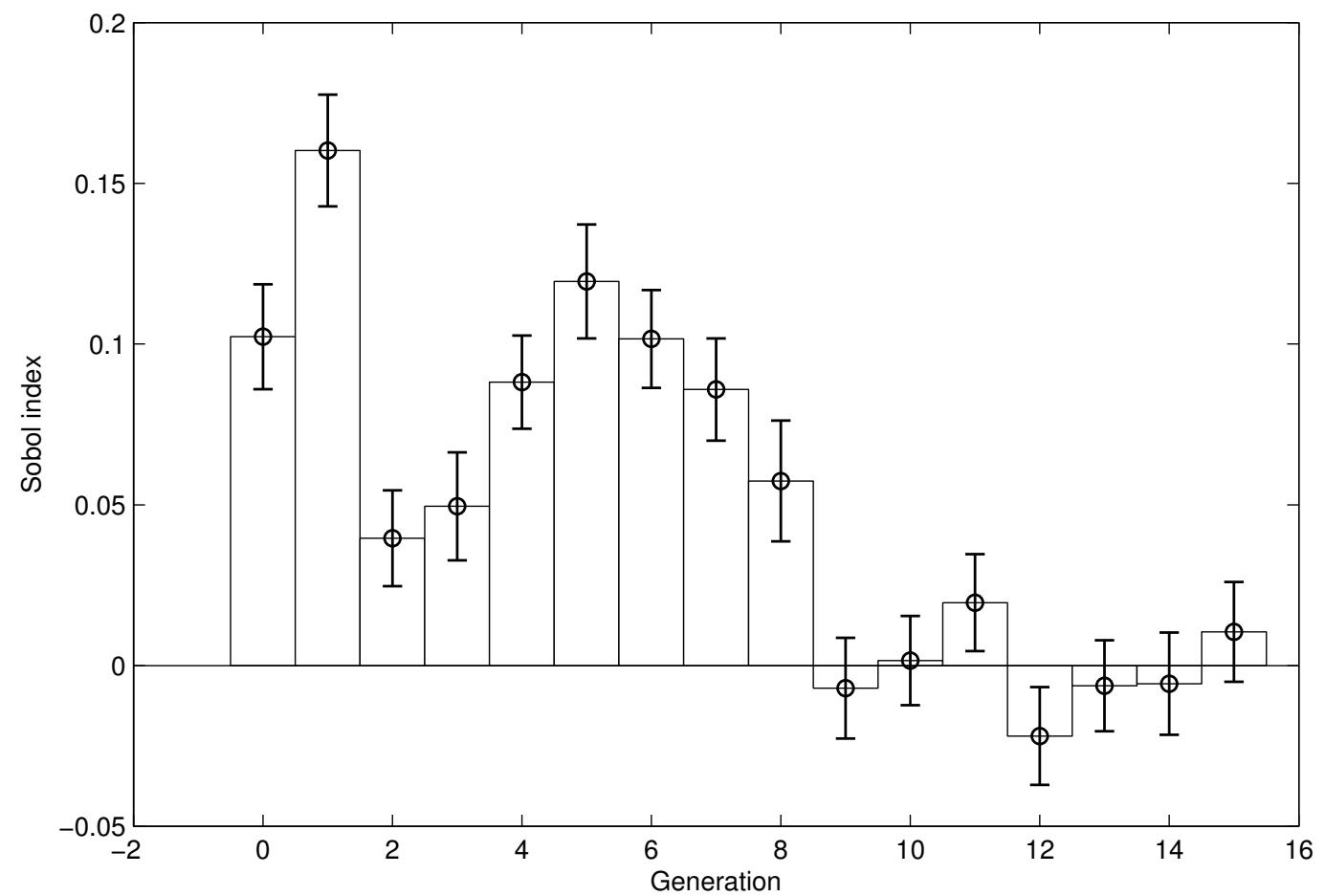

FIGURE 5. Sobol indices

(3) As for the respective contributions of the branches upon the global variability, Sobol analysis exhibits two distinct zones: a proximal one (branches 0 to 7 or 8 ), in which the contribution to global variability is fairly uniformly distributed over generations, and a distal one (generation 9 and above), that corresponds to generations which do not significantly contribute to variability.

All these arguments tend to support the relevance of the resistance as a lumped parameter. The respiratory tract as a resisitive network remains a complex system, but this complexity is in someway smoothed by nonlinear interactions between parameters, and the tree structure provides a great robustness to the overall process.

\section{REFERENCES}

[1] A. Ben-Tal, Simplified models for gas exchange in the human lungs, J. Theor. Biol. 238 (2006), 474-495.

[2] R. Carlson, Myopic models of population dynamics on infinite networks, Networks And Heterogenous Media, Volume 9, Number 3, September 2014.

[3] J.E. Cotes, D.J. Chinn, M.R. Miller, Lung Function, Blackwell 2006.

[4] F. Bernicot, B. Maury, D. Salort, A 2-adic approach of the human respiratory tree, Netw. Heterog. Media 5 (2010), no. 3, $405-422$.

[5] S.-Y. Chung, Y.-S. Chung, J.-H. Kim, Diffusion and Elastic Equations on Networks, Publ. RIMS, Kyoto Univ. 43 (2007), $699 ? 726$.

[6] P.G. Ciarlet, Introduction to Numerical Linear Algebra and Optimisation, Cambridge Texts in Applied Mathematics (Book 4), Cambridge University Press (1989).

[7] P. Doyle, J. L. Snell, Random Walks and Electric Networks, Mathematical Association of America, 1984.

[8] C. Grandmont, B. Maury and N. Meunier, A viscoelastic model with non-local damping application to the human lungs, ESAIM : M2AN, 40(1):201-224, January 2006. 
[9] R.H. Ingram, T.J. Pedley, Pressure-flow relationship in the Lungs, in Comprehensive Physiology, supplement 12: Handbook of Physiology, The respiratory system, mechanics of breathing, 2011, 277-293.

[10] A. Janon, T. Klein, A. Lagnoux, M. Nodet, C. Prieur, Asymptotic normality and efficiency of two Sobol index estimators, 2012. Accepted in ESAIM: Probability and Statistics.

[11] M. Kramar Fijavž, D. Mugnolo, E. Sikolya, Variational and Semigroup Methods for Waves and Diffusion in Networks, Appl Math Optim 55:219-240 (2007).

[12] B. Mauroy, N. Meunier, Optimal Poiseuille flow in a finite elastic dyadic tree, ESAIM : M2AN , 42, 507-534, July-August 2008.

[13] B. Mauroy, M. Filoche, E. R. Weibel, and B.Sapoval, An optimal bronchial tree may be dangerous, B. Mauroy, Nature, 427, 633-636, 12 February 2004.

[14] B. Mauroy, M. Filoche, J.S. Andrade Jr., B. Sapoval, Interplay between flow distribution and geometry in an airway tree, Phys. Rev. Lett. 90, 14 (2003).

[15] B. Mauroy and P. Bokov, Influence of variability on the optimal shape of a dichotomous airway tree branching asymmetrically, Phys. Biol., 7:016007 (2010).

[16] B. Maury, The Respiratory System in Equations, (MS\& A), ed. Springer, 2013.

[17] B. Maury, D. Salort, C. Vannier, Trace theorems for trees, application to the human lung, Network and Heterogeneous Media, Volume 4, Number 3, September 2009, 469-500.

[18] Pedley, T.J., R.C. Schroter, and M.F. Sudlow, The prediction of pressure drop and variation of resistance within the human bronchial airways, Respir Physiol, 1970. 9(3): p. 387-405.

[19] T. Ritz, B. Dahme, A.B. Dubois, H. Folgering, G.K. Fritz, A. Harver, H. Kotses, P.M. Lehrer, C. Ring, A. Steptoe, and K.P. Van de Woestijne, Guidelines for mechanical lung function measurements in psychophysiology, Psychophysiology, 39 (2002), 546-567.

[20] Fritz Rohrer, Der Strömungswiderstand in den menschlichen Atemwegen und der Einfluss der unregelmässigen Verzweigung des Bronchialsystems auf den Atmungsverlauf in verschiedenen Lungenbezirken, Pflüger's Archiv für die gesamte Physiologie des Menschen und der Tiere 28. Oktober 1915, Volume 162, Issue 5-6, pp 225-299.

[21] P. M. Soardi, Potential Theory On Infinite Networks, Springer-Verlag (1994).

[22] I.M. Sobol', Global sensitivity indices for nonlinear mathematical models and their Monte Carlo estimates, Mathematics and Computers in Simulation 55 (2001) 271-280.

[23] T.T. Soong, P. Nicolaides, C.P. Yu, S.C. Soong, A statistical description of the human tracheobronchial tree geometry, Respir Physiol. 1979 Jul;37(2):161-72.

[24] E.R. Weibel, Morphometry of the human lung, Springer Verlag and Academic Press, Berlin, New York, 1963.

[25] E.R. Weibel, The Pathway for Oxygen, Harvard University Press, 1984.

[26] I. Weinhold, G. Mlynski, Numerical simulation of airflow in the human nose, Eur Arch Otorhinolaryngol. 261:452-455, 2004.

[27] J. Wen, K. Inthavong, J. Tu, S. Wang, Numerical simulations for detailed airflow dynamics in a human nasal cavity Respir. Physiol. Neuro. 161:125-135, 2008.

[28] W. Woess, Dirichlet problem at infinity for harmonic functions on graphs, International Conference on Potential Theory 1994, Proceedings (editors: J. Kral et al.), de Gruyter, Berlin (1996) pp. 189-217. 